\title{
The Physiology of Substance P in the Rabbit Retina
}

\author{
Robert A. Zalutsky and Robert F. Miller \\ Washington University School of Medicine, Department of Ophthalmology, St. Louis, Missouri 63110
}

The neuropeptide substance $P$ (SP) has been localized to amacrine and ganglion cells in the rabbit retina. We have examined the effects of SP and related peptides on rabbit retinal neurons using bath application and intra- and extracellular electrophysiological methods in an in vitro retina eyecup preparation.

Substance $P$, at concentrations as low as 25 nM, moderately excited most brisk ganglion cells. SP excited some ganglion cells directly during cobalt block of synaptic transmission. Intracellular recordings from amacrine cells demonstrated that some, but not all, were depolarized by SP; pharmacological evidence suggested GABAergic amacrines were probably among those sensitive to SP. SP did not affect horizontal cells or the ERG, suggesting that the effects of this peptide are confined to the inner retina. The effects of SP were strongly potentiated by peptidase inhibitors, raising the possibility that endogenously released SP may act quite locally in the rabbit retina. The relative potencies of SP and the related peptides substance $K$ and eledoisin on different cells suggest that more than one tachykinin receptor subtype is present in the rabbit retina.

The responses of ganglion cells to SP desensitized with repeated or prolonged applications. Comparison of a cell's light responses before and after the receptors were desensitized revealed no qualitative changes in receptive field characteristics, but quantitative changes in excitability were apparent. SP antagonist analogs, although not potent, specifically blocked the effects of SP on some ganglion cells. The effects of these antagonists on light responses reinforced the inferences from desensitization paradigms regarding the role of endogenous SP.

The results of this study suggest that SP does not participate in shaping the characteristic receptive field properties of ganglion cells, but SP-or related peptides-may modulate the excitability of inner retinal neurons.

Substance $P$ (SP) is widely distributed in the ncrvous system (see Hökfelt et al., 1986) and has been strongly implicated as a transmitter in the CNS and periphery, based on the classical criteria for transmitter identification (Otsuka and Konishi, 1983,

\footnotetext{
Received Jan. 23, 1989; revised July 17, 1989; accepted July 18, 1989.

This research was supported by NEI grants EY03014 and EY07057.

Correspondence should be addressed to Robert A. Zalutsky at his present address: Department of Pharmacology, S-1210, University of California at San Francisco Medical School, San Francisco, CA 94143.

Reprint requests should be addressed to Robert F. Miller at his present address: Department of Physiology, University of Minnesota Medical School, 6-255 Millard Hall, Minneapolis, MN 55455.
}

Copyright (C) 1990 Society for Neuroscience $0270-6474 / 90 / 100394-09 \$ 02.00 / 0$ and Dun, 1985, for reviews). In the mammalian retina, the presence of SP has been demonstrated by both immunohistochemical and chromatographic methods (Famiglietti et al., 1980; Eskay et al., 1981; Brecha et al., 1984; Osborne, 1984); highaffinity SP binding and potassium evoked release of SP in a mammalian retina have also been reported (Osborne, 1984). In the rabbit retina, SP-like immunoreactivity is present in at least 2 types of amacrine cells (Famiglietti et al., 1980; Sagar et al., 1983; Brecha et al., 1984) and in ganglion cells (Brecha et al., 1985, 1987).

Despite the evidence suggesting a role for SP in neurotransmission in other parts of the nervous system, there has been no demonstration that SP has physiological effects consistent with such a role in the mammalian retina. In the carp retina (Glickman et al., 1982), SP applied by atomizer and in the mudpuppy retina SP applied iontophoretically (Dick and Miller, 1981) strongly excited most ganglion cells. In the mudpuppy retina, bath-applied SP at low micromolar concentration excited ganglion cells by a conductance increase mechanism (R. A. Zalutsky and $R$. F. Miller, unpublished observations), but in many retinas, no cells responded to SP. Since these cells had apparently normal light responses, SP cannot be crucial for driving those hasic responses. The greater consistency of $\mathrm{SP}$ effects in the mammalian (rabbit) compared with the amphibian (mudpuppy) retina greatly facilitated the more detailed analysis of SP actions presented in this study. Our results indicate that SP, at low concentrations, does affect inner retinal neurons, and endogenous SP may contribute to the regulation of ganglion cell excitability, but our results also indicate that SP does not play a crucial role in shaping the commonly recognized ganglion cell receptive field properties.

\section{Materials and Methods}

The methods of this study were identical to those described in the accompanying paper (Zalutsky and Miller, 1990), and similar to those which have been described in detail elsewhere (Miller et al., 1986). Briefly, SP and other drugs were bath-applied using an in vitro rabbit retina eyecup preparation. Light stimuli were presented by an optical bench, and neuronal responses were monitored using the transretinal electroretinogram (ERG), single-unit extracellular, and intracellular recording methods.

\section{Results}

\section{Excitation of ganglion cells}

Substance P was bath-applied to more than 240 ganglion cells in this study. In about $15 \%$ of recorded ganglion cells, SP produced strong excitations, that is, a $>100 \%$ increase in total spiking. Figure 1 shows one such example. The extent of the SP excitation is evident from the ratemeter record at the top and from the onset of the excitation in the raw spike record below.

The most common effect of SP at low concentrations $(<100$ 


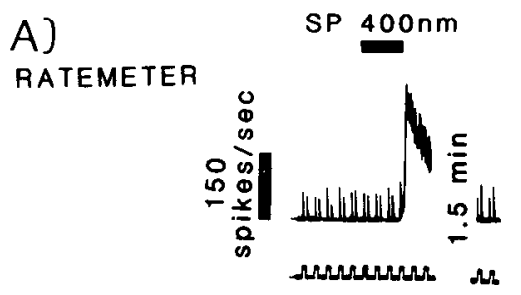

B)
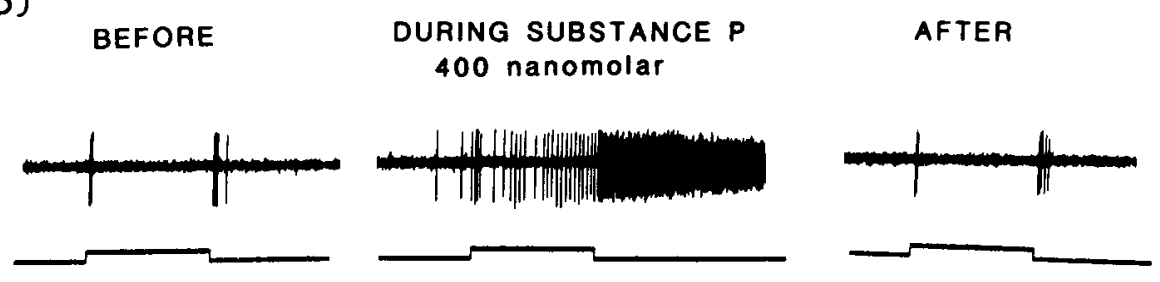

Figure 1. SP excitation of an On-Off direction-selective ganglion cell responding to a stationary flashing spot. $A$, Ratemeter records; $B$, individual light responses before, during, and after application of SP.

nM) and even at maximal concentrations $(>500 \mathrm{nM})$ on most cells $(60 \%$ of the total) was a moderate increase in spontaneous spiking with a comparatively smaller increase in light-evoked activity. Figure 2 shows 2 examples. In Figure $2 A$, a low concentration of SP (50 nM) and in Figure $2 B$ a maximal dose (500 $\mathrm{nM}$ ) on another cell had a much greater effect on the spontancous activity compared with its action on the light-evoked spiking, as the numbers in the figure indicate.

Approximately $20 \%$ of the cells examined showed no obvious change in spiking to SP, even at concentrations which produced maximal effects on other cells (>500 nM). However, in these cells and at subthreshold concentrations (on cells showing excitations to higher SP concentrations), SP potentiated the effects of other excitatory agents, as illustrated in Figure 3. In this example, SP alone had no obvious effect on this direction-selective cell. Likewise, the excitatory amino acid analog kainic acid, at a low dose $(5 \mu \mathrm{m})$, had no apparent effect. However, when the 2 agents were combined, a strong excitation occurred. While the mechanism of this synergistic effect is not clear from this experiment, it is apparent that SP did indeed have a demonstrable effect on the cell.

It is often stated that SP actions in the CNS are all quite slow, with latencics of a few seconds. If this were so in the retina, any participation of SP in shaping rapid retinal light responses could be immediately dismissed. However, the claims of slow SP action are based on iontophoretic experiments, and the iontophoresis of SP is itself a slow, limiting process (Guyenet et al., 1979). In the present experiments, the perfusion system was configured to allow comparison of the latencies of substances known to act quickly (e.g., excitatory amino acids) with the latency of SP on the same cells. Using such pairwise comparisons, the latencies could be measured to within about $500 \mathrm{msec}$ of each other. With this limitation in mind, we could detect no difference in latency between the SP excitation and that evoked
A) BHISK SUSTAINED CELL

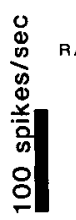

$$
\begin{aligned}
& \text { SUBSTANCE P } 50 \text { nanomolar } \\
& 30 \text { sec }
\end{aligned}
$$

CONIROL

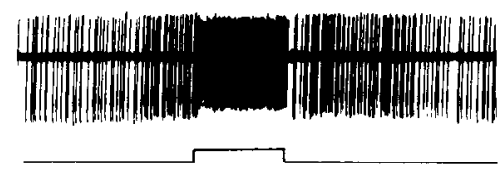

light evoked 131 spontaneous 125

DURING SUBSTANCE $P$

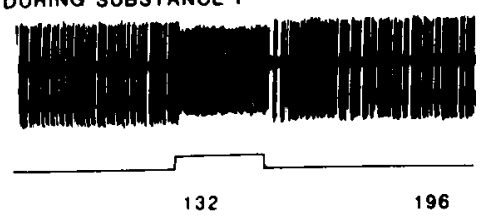

B) BRISK TRANSIENT OFF CELL

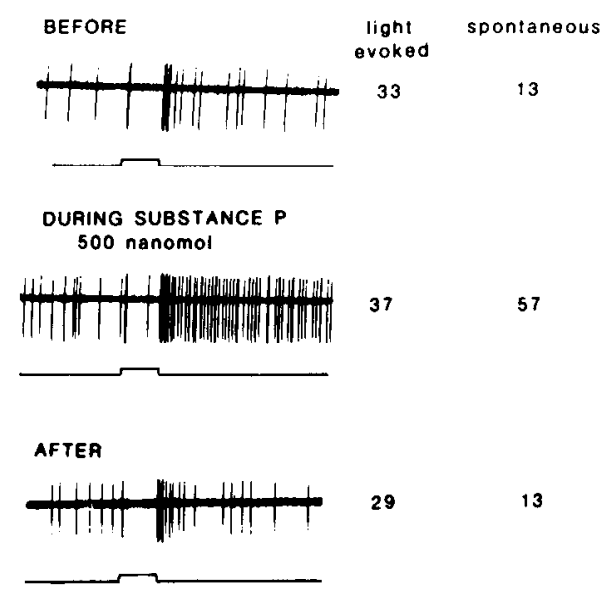

Figure 2. SP excitations of an On ganglion cell $(A)$ and an Off cell $(B)$. The cell in $A$ responding to a low dose (50 nM) and the cell in $B$ responding to a maximal dose $(500 \mathrm{nM})$ both show much greater increases in spontaneous activity than light response as the numbers indicate. Scale bar (in $A$ ), 100 spikes/ sec. 

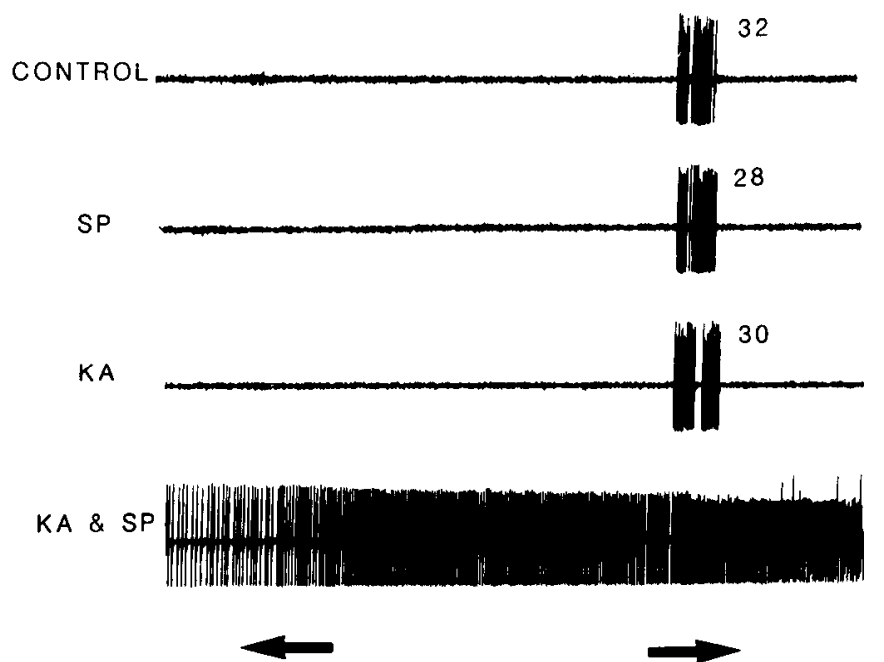

Figure 3. SP potentiation of excitatory amino acid excitation. SP even at the high concentration $1 \mu \mathrm{M}$ had no effect on this On-Off directionselective cell. The excitatory amino acid agonist kainate at the low concentration of $5 \mu \mathrm{M}$ also had no effect. When the 2 were combined, a substantial excitation resulted, indicating that SP did affect the cell. The arrows indicate the direction of movement of the $100 \mu \mathrm{M}$ slit stimulus.

by excitatory amino acids (glutamate or kainate). In contrast, using the same methods, the latency of action of another peptide, somatostatin (Zalutsky and Miller, 1990) was clearly slower than that of conventional transmitters and SP. Thus, we categorically reject the notion that SP actions are unavoidably slow in onset.

SP was applied to more than 240 ganglion cells in this study. Since the response of a cell was critically dependent on the general status of the retina and upon previous exposure to SP (discussed below), Table 1 was compiled from retinas which were unquestionably healthy and which had not previously been exposed to SP or other peptides. A "healthy" retina was characterized by large b- and c-waves in the ERG and sharply tuned ganglion cell light responses. Table 1 shows the numbers and percentages of cells which increased spiking at least $20 \%$ to SP at the indicated concentrations. Table 1 shows that the percentage of cells responding to SP did not increase as the concentration of the peptide was elevated above $500 \mathrm{~nm}$. Multiple concentrations of SP were applied to more than 80 cells in an attempt to determine the threshold and maximal doses. Unfortunately, the problems of desensitization, the variability of maximal responses among different cells, and the interaction of

\begin{tabular}{|c|c|c|}
\hline Concentration & $\begin{array}{l}\text { Cells excited } \\
\text { total cells }\end{array}$ & $\%$ Excited \\
\hline$<1 \mu \mathrm{M}$ & $22 / 28$ & 78 \\
\hline$<500 \mathrm{nM}$ & $40 / 49$ & 82 \\
\hline$<250 \mathrm{nM}$ & $31 / 41$ & 76 \\
\hline$<125 \mathrm{nM}$ & $36 / 65$ & 55 \\
\hline
\end{tabular}

Numbers and percentages of ganglion cells showing at least a $20 \%$ increase in spiking to various concentrations of SP. The concentration categories are not cumulative; that is, $<15 \mu \mathrm{M}$ indicates $<1 \mu \mathrm{M}$ but $>500 \mu \mathrm{M}$, the next category. Because the response of a cell depends strongly on the health of the retina and on previous exposure to $\mathrm{SP}$, this table includes only those cells whose responses were not confounded by those variables.
A.
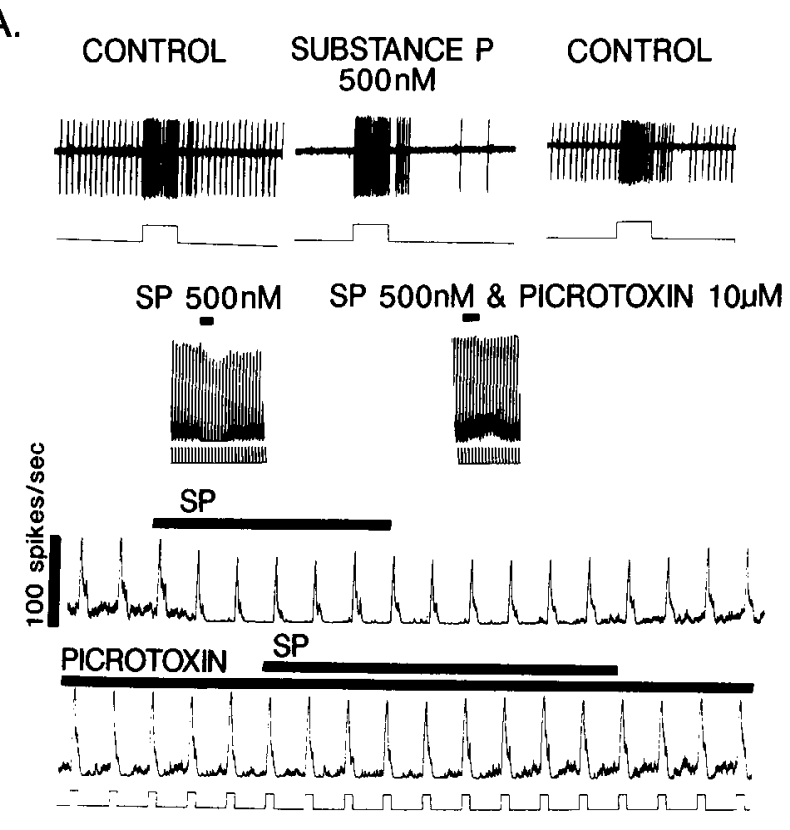

B.

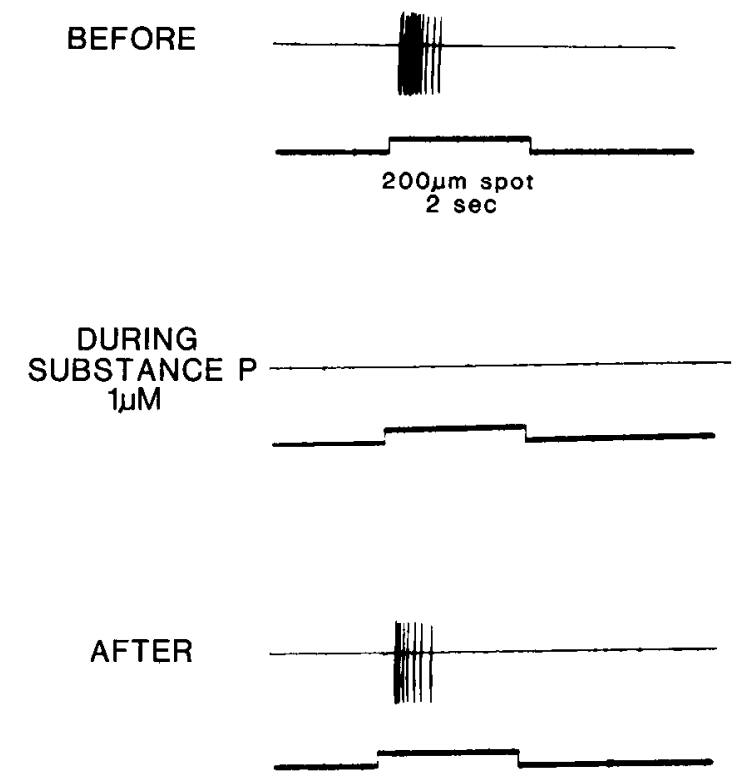

Figure 4. A, SP inhibitions of brisk ganglion cells in unhealthy retinas were blocked by picrotoxin, suggesting the inhibitions resulted from SP excitation of GABAergic amacrines. $A$, Ratemeter records in the presence and absence of picrotoxin. $B$, Extracellular recording from a sluggish On cell showing a SP inhibition. The cell was classified as sluggish because it showed neither spontaneous activity nor response to a fullfield stimulus, responded to only very slowly moving stimuli, and had a low maximal light-stimulated spiking rate.

direct and indirect effects at the ganglion cell level precluded any determination of meaningful dose-response curves. (Each of these issues is discussed further below.) However, in general, the saturating concentration was clearly below $500 \mathrm{~nm}$ in at least $90 \%$ of the cells examined and $<250 \mathrm{nM}$ in at least $50 \%$ of the cells. The threshold concentration was most easily determined in cells with significant levels of spontaneous activity; in these cells effects of SP were generally first evident at concentrations of about $25 \mathrm{~nm}$. 

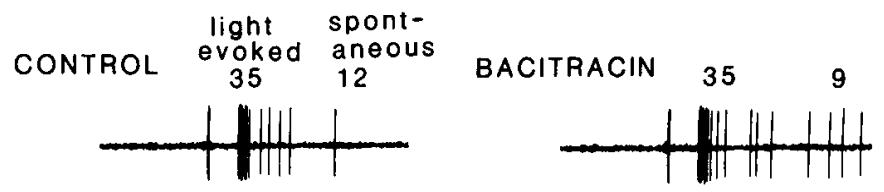

SP

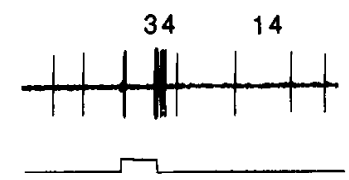

BACITRACIN \& SP

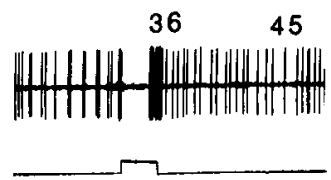

Figure 5. Extracellular recording from an Off ganglion cell showing SP $(100 \mathrm{nM})$ excitations in the presence and absence of the peptidasc inhibitor bacitracin $(100 \mu \mathrm{M})$. The numbers indicate light-evoked and spontaneous spikes per stimulus. The stimulus traces indicate $1 \mathrm{sec}$ large spot stimuli.

\section{Substance $P$ inhibitions are indirect}

Substance $\mathbf{P}$ inhibited some brisk ganglion cells. Such inhibitions were substantially blocked by low micromolar doses of picrotoxin (Fig. 4A). At these concentrations, picrotoxin is demonstrably a specific GABA antagonist in this preparation (i.e., blocks GABA but not glycine inhibition). This and evidence presented below suggest that the SP inhibitions result from SP activation of GABAergic amacrines, which then inhibit ganglion cells. Intracellular recordings from amacrine cells (see below) are consistent with this possibility.

\section{Ganglion cell receptive field types and SP actions}

Ganglion cell receptive field types were classified according to criteria based on Caldwell and Daw (1978). Light stimuli included small spots, annuli, full-field dimming, and slits with variable orientations, speed, and direction of movement. SP excited all brisk ganglion cell types, including On and Off center, sustained and transient, large field units, and On-Off directionselective cells. However, even in unquestionably healthy retinas, brisk cells of the same receptive field type varied greatly in their maximum response to SP. For example, the cell in Figure 1 which was strongly excited by SP, and the cell in Figure 3, which showed no response to the same maximal dose, were both unambiguously identified as On-Off direction-selective cells. In contrast, the responses of cells to conventional transmitter agonists were much better correlated with receptive field type. For example, all On-Off direction-selective cells were strongly excited by low doses of cholinergic agonists (as expected from Aricl and Daw, 1982).

A small number of sluggish cells were encountered in this study. In vivo experiments have demonstrated that the slower conduction velocity of sluggish cells serves to corroborate their identification (Caldwell and Daw, 1978; Vaney et al., 1981). In these in vitro experiments, this was not feasible. Three of 10 apparent sluggish cells were inhibited by SP (e.g., Fig. 4B), 2 were slightly excited, and the rest showed no apparent response to SP. On direction-selective cells have characteristics somewhat similar to sluggish cells and are unambiguously identifiable; about a third of these were inhibited by SP, and none were strongly excited. As discussed above, the inhibitions probably reflect SP excitation of GABAergic amacrine cells. The difference between brisk and sluggish cell responses to SP may reflect
A.

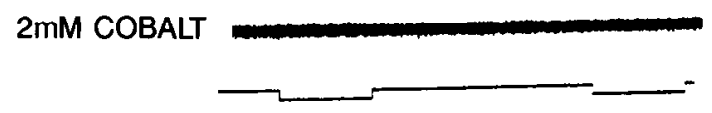

SUBSTANCE P $2 \mu \mathrm{m}$

2mM ${ }^{\text {in }}$ COBALT

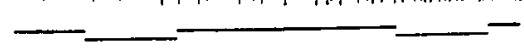

B.
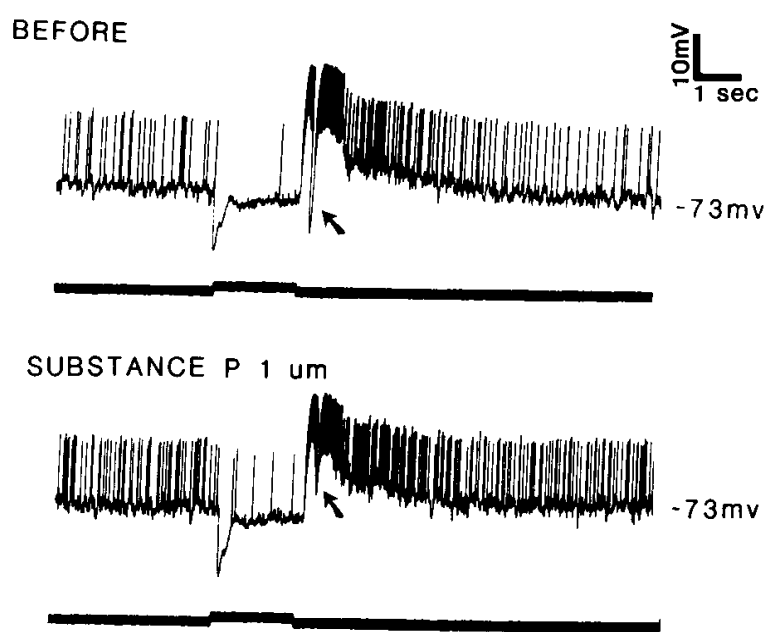

AFTER

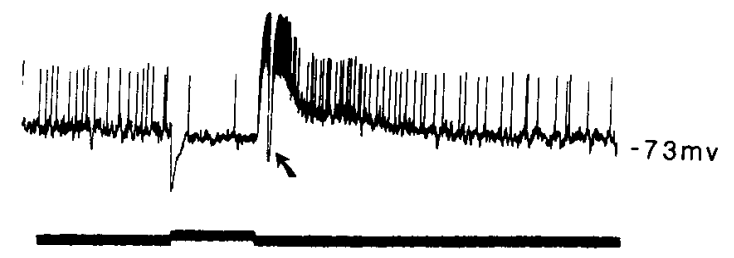

Figure 6. A, Extracellular recordings showing SP excitations during cobalt block of synaptic transmission. $B$, Intracellular recordings from an Off ganglion cell before, during, and after SP. Spontaneous spiking increased during SP with no apparent depolarization. Note the reduced inhibitory input at the arrow. This probably reflects decreased input from an inhibitory amacrine cell and reinforces the idea that SP effects in the inner retina are very complex; the spikes were attenuated by the chart recorder.

a greater dominance of inhibitory ovcr excitatory inputs in sluggish cells, as suggested by their receptive field properties.

The sensitivity to SP was approximately equal in all areas of the retina, including superior and inferior, center and periphery, and within and outside of the visual streak. The distribution of SP immunoreactivity among these areas has not been reported in the rabbit retina but does vary in some nonmammalian species (Brecha et al., 1984).

\section{Degradation of $S P$}

In peripheral ganglia, some peptides are degraded slowly enough that they can diffuse and act at a considerable distance from the sites of release (see Jan and Jan, 1982). There is suggestive evidence that this may be so for somatostatin in the rabbit retina 
A.

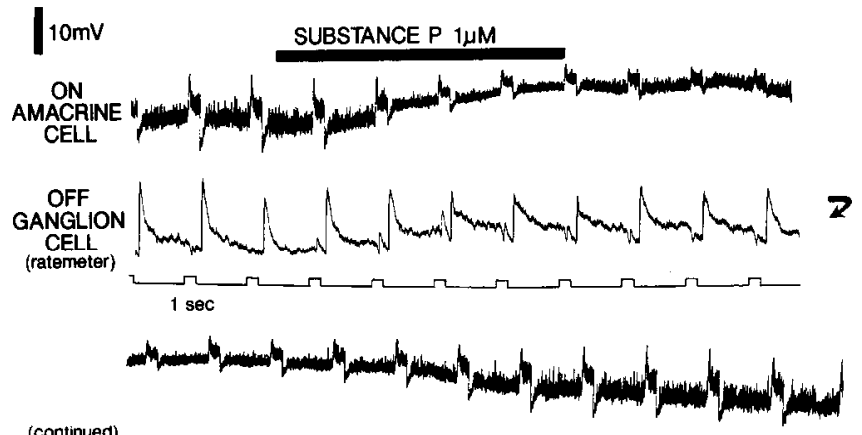

(continued)

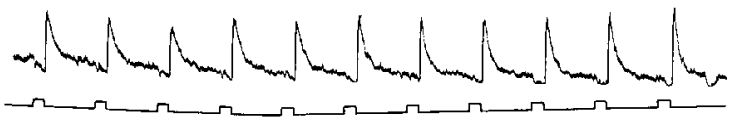

B.

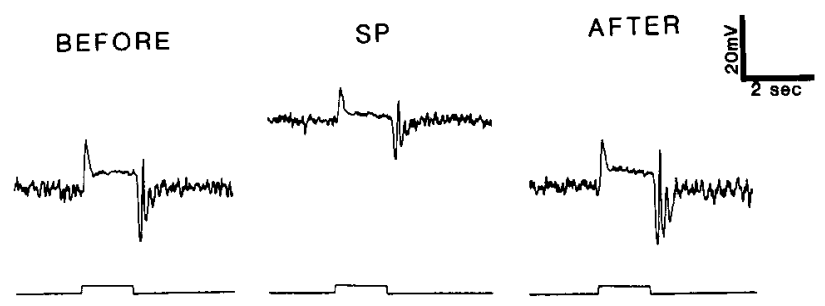

Figure 7. A, Intracellular recording from On amacrine cell (probably an A-II amacrine) and ratemeter record from simultaneous extracellular recording of Off ganglion cell during SP application. SP depolarized the amacrine cell by about $10 \mathrm{mV}$ from its resting potential of $-74 \mathrm{mV}$ and reduced the synaptic noise substantially, suggesting a conductance increase. The ganglion cell spontaneous activity was increased with a similar time course. $B$, Intracellular records from an On amacrine (also probably an A-II amacrine) cell before, during, and after application of $250 \mathrm{~nm}$ SP. SP depolarized the cell and decreased the amplitude of all components of the light response, suggesting a conductance increase. The dark resting potential was $-55 \mathrm{mV}$.

(Zalutsky and Miller, 1990). We examined whether degradation strongly limited SP actions in the retina by comparing the potency of SP in the presence and absence of the peptidase inhibitor bacitracin. This inhibitor is not specific for SP degradation, but the concentration dependence of its effect on the degradation of endogenously released SP in mammalian CNS has been well described (Mauborgne et al., 1987). Figure 5 illustrates that bacitracin substantially potentiated SP effects at a sufficiently low concentration that bacitracin itself had no effect. Since inhibition of peptidases potentiates the effects of applied SP, the diffusion of endogenously released SP must also be limited by peptidase activity. It is not possible to infer whether SP actions are confined to a single synapse, but apparently this peptide cannot diffuse as freely as peptides inn the periphery or somatostatin in the rabbit retina.

\section{Sites of SP action}

We studied the sites at which SP acts in the retinal network by using cobalt to block synaptic transmission and intracellular recordings obtained from ganglion cells and other retinal neurons.

SP had clear direct effects on some ganglion cells, observed during cobalt block of synaptic transmission. Figure $6 A$ shows SP excitation of an extracellularly recorded ganglion cell during cobalt block of synaptic transmission. Intracellular recordings from ganglion cells sometimes showed a small depolarization associated with an increased rate of firing, but in some instances, such as that illustrated in Figure $6 B$, an increase in firing rate was detected in the presence of SP without any clear change in the dark membrane potential of the cell. In addition, Figure $6 B$ illustrates a second, more complex action of SP: In this example, SP application reduced the magnitude of an IPSP evoked at light off and is indicated by the arrows. This observation points to network effects of SP which are presynaptic to the ganglion cell or which modulate different types of synaptic receptors at the level of the ganglion cell. Intracellular recordings from amacrine cells (Fig. 7) indicate that SP depolarization of amacrine cells could contribute to the loss or reduction of amacrine cellmediated responses seen in ganglion cells.

Some, but not all, amacrine cells were depolarized by SP (17 of 28), examples of which are illustrated in Figure 7, which shows recordings that are probably from A-II amacrines, although this identification was not unambiguously established with cell staining. The depolarizations in amacrine cells were generally less than $10 \mathrm{mV}$, which was substantially less than that observed when excitatory amino acids were applied to the same cells. The amplitude of both positive- and negative-going components of the light responses and of the synaptic noise were diminished during SP depolarizations (Fig. $7 B$ ), suggesting a conductance increase mechanism. Unfortunately, attempts to measure this conductance change directly, with current pulses

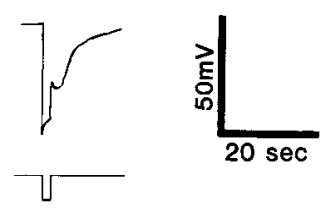

Figure 8. The absence of effect of SP $(1 \mu \mathrm{M})$ on a horizontal cell. Top trace, the maximum response of the cell to bright light (dark resting potential, -32 $\mathrm{mV}$ ). The stimuli below are small spot and full field.

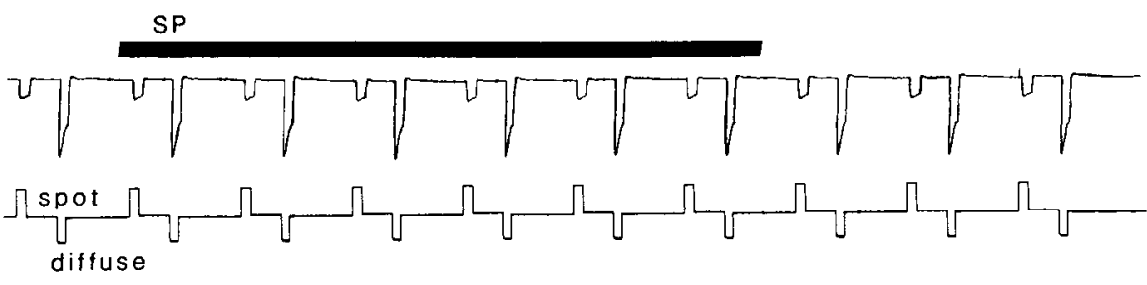


A.

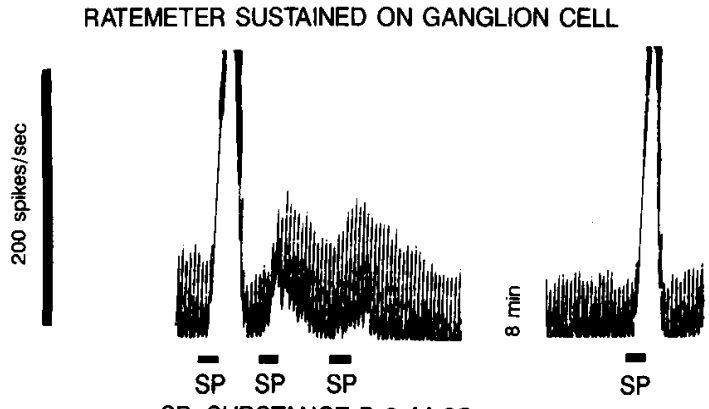

SP: SUBSTANCE P $2 \mu \mathrm{M} 25 \mathrm{sec}$

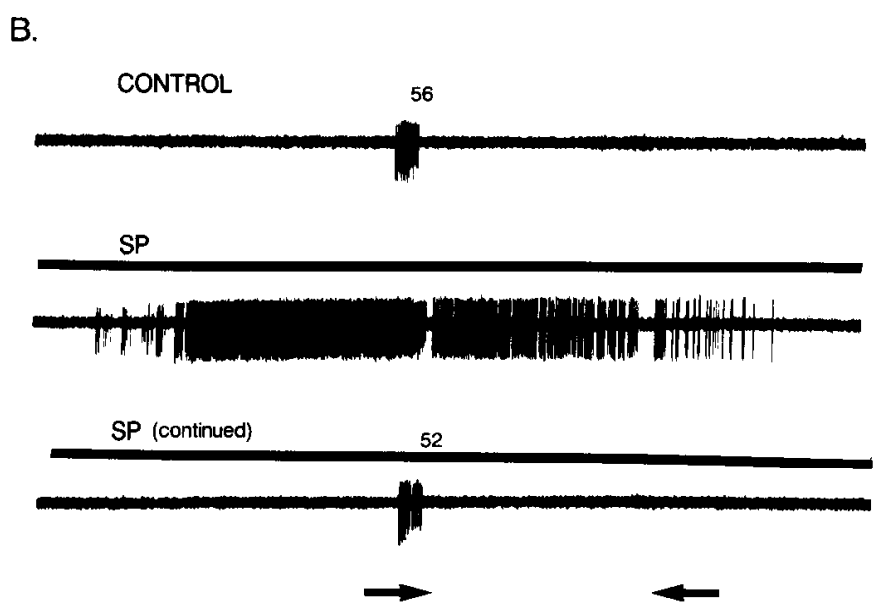

Figure 9. A, Ratemeter records from an On ganglion cell showing SP desensitization. Each upward deflection of the ratemeter indicates the On response to a $2 \mathrm{sec}$ stimulus at this compressed time scale. Three successive applications of SP $(2 \mu \mathrm{M})$ produced progressively reduced excitations. A final application after about $10 \mathrm{~min}$ showed the recovery of sensitivity to SP. $B$, Effects of SP desensitization on a directionselective cell. The arrows indicate the directions of movement of a 100 $\mu \mathrm{M}$ slit of light. The numbers indicate the average numbers of spikes per stimulus presentation. A continuous application of SP $(1 \mu \mathrm{M})$, indicated by the dark bar, produced an excitation which desensitized. In the continued presence of SP, the desensitized cell (lowest trace) was still direction selective and showed a small but consistent reduction in spiking.

injected through the intracellular recording pipette, produced ambiguous results regarding the conductance change, possibly reflecting a more complex mechanism or simply the technical difticulties of making such determinations with the high-resistance electrodes necessary to impale such small cells.

$\mathrm{SP}$, even at micromolar concentrations, had no apparent effect on horizontal cells, as illustrated in Figure 8. SP had only very small effects on the b-wave of the ERG: We observed both increases and decreases in ERG amplitude. This is consistent with the effects of this peptide being restricted to amacrine and ganglion cells and probably rules out any strong effect of SP action at the level of On bipolar cells.

\section{Desensitization of $S P$ receptors}

The excitatory actions of SP strongly desensitized. After a single application at high concentrations (micromolar), or repeated or prolonged applications at more moderate concentrations, the response to SP was usually dramatically reduced. Lower concentrations $(<100 \mathrm{~nm})$ did not rapidly desensitize. Figure 9
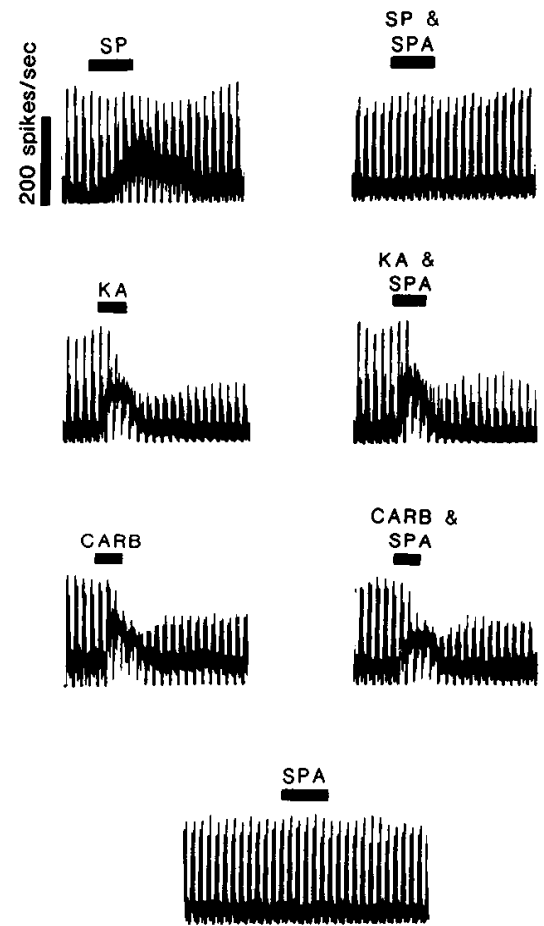

Figure 10. Ratemeter records at a compressed time scale showing the specificity of SPA in blocking SP. The top row shows that $500 \mathrm{~nm}$ SP strongly excited the cell and $8 \mu \mathrm{M}$ SPA blocked this excitation. The middle rows show that SPA did not block cxcitations produced by the excitatory amino acid agonist kainic acid $(K A)$ or the cholinergic agonist carbachol $(C A R B)$. The bottom row shows that SPA alone had no apparent effect on this cell. The drug applications were spaced widely enough to avoid desensitization.

shows examples of desensitization to repeated doses and a single application of a high SP concentration. Cells were still responsive to other excitatory agents while nonresponsive to SP, suggesting desensitization of SP receptors as the mechanism.

The phenomenon of desensitization is useful for inferring the role of endogenous SP in retinal function. First, of course, SP obviously does not drive any prolonged light responses or responses which repeat many times without diminishing. More importantly, if receptors are desensitized to applied SP, they must also be insensitive to endogenous SP. By comparing the light responses of retinal neurons before and after desensitization, the contribution of SP to those responses can be inferred.

The effects of SP desensitization on ganglion cell light responses were carefully monitored in more than 20 cells, and more casually examined in many more in the course of other experiments. None of the fundamental receptive field properties of ganglion cells was changed while a cell was insensitive to SP, nor were the relative strengths of center and surround altered. Figure $9 B$ shows, for example, the responses of a directionselective cell before and after desensitization to a high concentration of SP; the arrows indicate the direction of a moving bar stimulus. While the overall receptive field character was unchanged during SP desensitization, most cells with spontaneous discharge showed a decrease (up to $30 \%$ ) in spontaneous activity associated with a smaller fraction of a reduced light response; the direction-selective cell in Figure $9 B$ showed a small but consistent decrease in light responses, as indicated by the average spike numbers. 

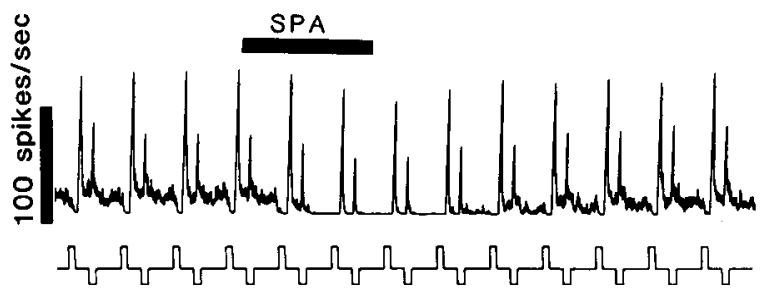

CONTROL

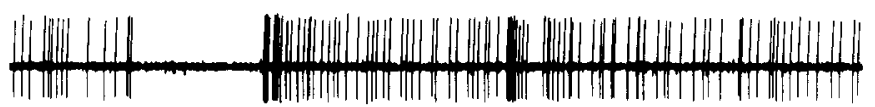

SPA
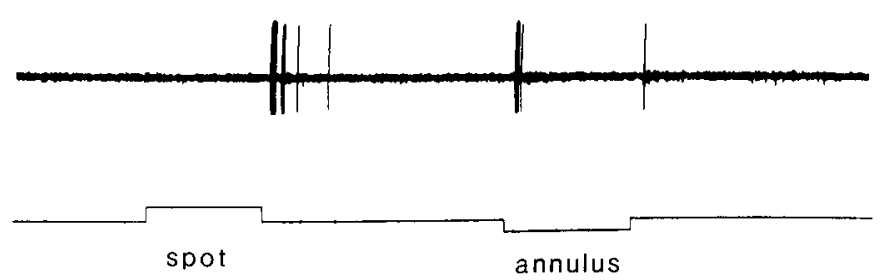

Figure 11. Ratemeter and individual light responses showing the effect of SPA $(10 \mu \mathrm{M})$ on an Off ganglion cell. Note that the light response was slightly reduced, the spontaneous activity was more strongly decreased, and the effect rapidly reversed.

\section{SP antagonists}

No universally potent and specific SP antagonist is available, but a number of analogs have proved useful in different locations (Hakanson and Sundler, 1985). Two analogs proved useful as antagonists in the rabbit retina: [d-arg1, d-pro2, d-trp7,9, leu 11] SP and [d-arg1, d-trp7,9, leu11] SP. The 2 had qualitatively identical actions in the rabbit retina, so are both referred to here as "SPA."

Figure 10 shows ratemeter records demonstrating that SPA blocked SP with useful specificity. The first row shows that 8 $\mu \mathrm{M}$ SPA blocked the excitation produced by a high SP concentration ( $500 \mathrm{~nm}$ ). The next two rows demonstrate that SPA did not block excitations produced by excitatory amino acid (here, kainic acid) or cholinergic (carbachol) agonists, which are the principal known excitatory transmitters in the rabbit retina. The final panel shows that SPA alone had no apparent effect on this cell's light responses. Another indication that SPA acted specifically was that when SP inhibited cells, SPA also blocked the inhibition.

The lack of effect of SPA on the light responses in Figure 10 was useful as a confirmation of the antagonist's specificity. In most cases (13 of 18), SPA did have slight effects on light responses similar to the effects of desensitization reported above. That is, SPA did not alter the defining characteristics of a cell's receptive field properties, but did slightly reduce spontaneous activity and, to a lesser extent, light responses. Figure 11 shows an example of the effects of SPA on an Off ganglion cell. In this example, SPA clearly diminished spontaneous firing and diminished, but comparatively less so, the light-evoked activity.

One of the SP antagonists used here, [d-arg1, d-pro2, d-trp7,9, leu 11] SP, has been reported to interact weakly with other peptide receptors, particularly bombesin, in some systems (Jensen ct al., 1984). 'The other antagonist, [d-arg 1, d-trp7,9, lcu 11 1] SP, is slightly less potent but reportedly does not interact with these peptides. Bombesin does have effects in the rabbit retina (Zalutsky and Miller, unpublished observations), but the effects of SPA could not be explained by blocking bombesin. In particular, bombesin excitations were slow and long-lasting, while the effects of SPA reversed quickly (e.g., Fig. 11). Furthermore, bombesin inhibited some cells which were excited by SP and inhibited by SPA. Finally, the effects of the 2 antagonists in the rabbit retina were qualitatively similar. The possibility that SPA is interacting with some unknown peptide cannot be dismissed. Nonetheless, whatever else these antagonists did, they clearly blocked SP actions, so the conclusion that SP cannot be a major drive for the dominant light responses remains valid. Equally valid, however, is the idea that tachykinins contribute to the excitability, particularly the spontaneous firing of retinal ganglion cells.

In order to avoid possible nonspecific effects, concentrations of SPA greater than $10 \mu \mathrm{M}$ were not used in our study. These concentrations were not always adequate to effectively block SP. The ability of SPA to block SP and the specificity of the antagonism were tested before the effects of SPA on light responses were examined in each cell. Some of the variability in the potency of these antagonists probably reflects differential preference for tachykinin receptor subtypes (discussed below).

\section{Tachykinin receptor subtypes}

$\mathrm{SP}$ is a member of the tachykinin family of peptides. At least 3 tachykinin peptides and at least 3 tachykinin receptor types are present in mammalian CNS (see Buck and Burcher, 1986, for review), though the situation is not yet certain for the retina. $\mathrm{SP}$ can bind to all receptor types, but the NK-1 (or $\mathrm{P}$ type), NK-2 (or K type), and NK-3 (or E type) prefer SP, substance $\mathrm{K}$ (SK), and eledoisin (ELE), respectively. We therefore compared the effects of SP, SK, and ELE on rabbit retinal ganglion cells.

SK and ELE excited some ganglion cells at concentrations as low as 10 nM. Below 125 nM, SK excited 9 of 15 and ELE 13 of 18 cells. Each of these tachykinins was dramatically more potent than SP in at least some cases. To further investigate receptor subtypes, the orders of potency of SP, SK, ELE, and, in some cases, SP methyl ester (which is more selective for the NK-1 receptor than SP itself) were determined on individual cells. Because of desensitization problems and the relatively weak maximal effects of tachykinins on many cells, reliable orders of potency were difficult to obtain; results were determined for 15 cells, focusing mainly on large-field Off units (8) and sustained On cells (4). Clear examples in which each of the 3 peptides was the most potent were observed in this sample. There was no apparent association of deduced tachykinin receptor subtype with ganglion cell receptive-field type; that is, cells with the same receptive field type clearly showed different orders of potency.

In a few cells, cross-desensitization experiments were attempted. The one clear result from these experiments was that sufficient concentrations of SP (500 nM) desensitized responses to the other tachykinins $(n=3)$. Therefore, SP at these concentrations can interact with all receptor types, as expected.

Other methods will be required to resolve the roles of the tachykinin receptor types. However, clearly more than one type is operative in the rabbit retina (from the orders of potency); $\mathrm{SP}$ at sufficient concentrations can interact with all of them 
(cross-desensitization), so the effects of SP must be interpreted accordingly; and the receptor types do not sort according to recognized ganglion cell receptive-field types as clearly as do conventional transmitters.

\section{Discussion}

SP at low concentrations excited brisk ganglion cells and depolarized some amacrine cells in the rabbit retina. Picrotoxin experiments suggested that GABAergic amacrines were among those affected by this peptide. The lack of sensitivity to SP among horizontal cells and the absence of effects on the ERG suggest that any functional role of endogenous SP is confined to the inner retina. In accord with this conclusion, experiments with peptidase inhibitors suggest that SP may be degraded too quickly to diffuse far from its sites of release, though whether its actions are confined to single synapses cannot be determined by these methods.

Desensitization paradigms and antagonist experiments present a consistent picture of the possible functions of endogenous SP. The most important conclusion that can be derived from the use of SP antagonists, or the act of desensitizing SP receptors, is that SP does not provide the drive for the fundamental, defining characteristics of ganglion cell receptive fields. That is, the polarity of the response (i.e., On vs Off), the sustained or transient nature of the discharge, the balance of center and surround, and the specialized response properties such as direction and motion sensitivity and the dimming response of large-field units were not substantially changed when cells are profoundly desensitized to SP. The same comparisons, however, do reveal a quantitative contribution of endogenous SP to the excitability of many ganglion cells. In particular, it appears that a portion of the spontaneous firing of many ganglion cells and, to a lesser extent, a small fraction of the excitatory response to light may be modulated directly or indirectly by SP. This result was obtained with both desensitization experiments and the application of SP antagonists. Thus, a role for SP in inner retinal function is strongly supported by our findings.

\section{Substance $P$ receptor subtypes and variability}

A study of the rank order of potency of the tachykinins SP, ELE, and SK demonstrates that more than one tachykinin receptor subtype must be present in the rabbit retina. Tachykinin receptor subtypes do not appear to distribute among ganglion cells according to receptive field type. Similarly, the sensitivity to SP among cells of the same receptive field type varies much more than the sensitivity to conventional transmitters. This supports the conclusion from desensitization and antagonist experiments that SP does not shape the characteristic receptive field properties of ganglion cells. Variability in peptide effects in general and SP, in particular (e.g., Jones, 1985), has been commonly observed, though not always emphasized. In this study, we have established that variability remains even when comparing cells of the same receptive field type, in similar retinal positions, in unquestionably healthy retinas, under identical lighting conditions. Just what variable(s) will explain the distribution of SP sensitivity and receptor types is obviously crucial for understanding the adaptive function of this peptide.

\section{Is SP a transmitter of ganglion cells?}

Recent evidence (Brecha et al., 1987) suggests that SP (or perhaps some other tachykinin) is found in ganglion cells of the rabbit retina and may be utilized by them at their axonal ter- minations. If this applies to the more common ganglion cell types we have examined in this study, then it is apparent that ganglion cells, which may synthesize and utilize tachykinins, are also sensitive to this peptide family when acting as a neurotransmitter. The significance of this dual role for SP in ganglion cell function awaits further analysis of the ganglion cell subtypes which contain SP. For example, if SP is localized to the sluggish ganglion cells, then it is possible that these neurons may synthesize and utilize SP but lack sensitivity to SP as a transmitter. Of course, the presence of a transmitter or modulator within a cell does not mean that the cell need be insensitive to the same agent acting as a neurotransmitter, since we are now quite adjusted to the concept of "autoreceptors" and the regulatory role such receptors might play in transmitter function.

\section{Comparison of SP and somatostatin in the rabbit retina}

Scveral ncuropcptides arc prescnt in the rabbit retina (Brccha et al., 1984) and several can affect retinal cells at reasonable concentrations (Zalutsky and Miller, unpublished observations). The actions of 2 peptides, SP and somatostatin (Zalutsky and Miller, 1990), have been examined in some detail in the rabbit retina, and it is useful to compare their actions.

Somatostatin apparently can act at multiple levels of the retinal network, and these actions converge to produce slow-onset, long-lasting adjustments of ganglion cell receptive-field properties. Consistent with this broad domain of action, experiments with a degradation-resistant somatostatin analog suggest somatostatin may be degraded slowly enough that it can act a distance from its sites of release. The cellular mechanism of somatostatin actions involves a conductance decrease. These results together suggest somatostatin might be conveniently classified as a paracrine modulator of the retinal network.

In contrast, SP, as described in the present study, acts exclusively in the inner retina and more conventionally in terms of space, time, and possibly mechanism. However, its role in the retinal neurocircuitry may not be so conventional, in that it does not shape ganglion cell receptive-field properties per se though it probably does contribute to excitability, in general, and to determining levels of spontaneous activity, in particular.

$A$ better understanding of the function of both SP and somatostatin in the retinal network will require a more detailed understanding of the microcircuitry of the rabbit retina and of the cellular mechanisms of the peptides' actions on retinal cells. Finally, while both SP and somatostatin may be "first messengers" in the retina, additional "hormonal" roles, as elsewhere demonstrated for these peptides, should not be dismissed.

\section{References}

Ariel, M., and N. W. Daw (1982) Effects of cholinergic drugs on receptive field properties of rabbit retinal ganglion cells. J. Physiol. (Lond.) 324: 135-160.

Brecha, N. C., W. Eldred, R. O. Kuljis, and H. J. Karten (1984) Identification and localization of biologically active peptides in the vertebrate retina. Prog. Retinal Res. 4: 185-226.

Brecha, N., M. Cillufo, and J. Bolz (1985) Identification of substance $\mathrm{P}$ immunoreactive ganglion cells in the rabbit retina. Soc. Neurosci. Abstr. 11: 1219.

Brecha, N., A. Lieberman, and J. Parnavelas (1987) Immunocytochemical evidence that substance $P$ may be a transmitter or neuromodulator at rabbit retino-geniculate synapses. J. Physiol. (Lond.) 382: P117.

Buck, S. H., and E. Burcher (1986) The tachykinins: A family with a brood of receptors. Trends Pharmacol. Sci. 7: 65-68.

Caldwell, J. H., and N. W. Daw (1978) New properties of rabbit retinal ganglion cells. J. Physiol. (Lond.) 276: 257-276. 
Dick, E., and R. F. Miller (1981) Peptides influence retinal ganglion cells. Neurosci. Lett. 26: 131-135.

Dun, N. J. (1985) Substance P. In Neurotransmitter Actions in the Vertebrate Nervous System, M. A. Rogawski and J. L. Barker, eds., pp. 385-410, Plenum, New York.

Eskay, R. L., J. F. Furness, and R. T. Long (1981) Substance P activity in the bullfrog retina: Localization and identification in several species. Science 212: 1049-1051.

Famiglietti, Jr., E. V., N. C. Brecha, and H. J. Karten (1980) Neural localization of substance $P$ like immunoreactivity in the rabbit retina. Soc. Neurosci. Abstr. 6: 212.

Glickman, R. D., A. R. Adolph, and J. E. Dowling (1982) Inner plexiform circuits in the carp retina: Effects of cholinergic agonists, GABA and substance $P$ on the ganglion cells. Brain Res. 234: 81-99.

Guyenet, P. G., E. A. Mroz, G. K. Aghajanian, and S. E. Leeman (1979) Delayed iontophoretic ejection of substance $P$ from glass micropipettes: Correlation with the time course of neuronal excitation in vivo. Neuropharmacology 18: 553-558.

Hakanson, R., and F. Sundler, eds. (1985) Tachykinin Antagonists, Elsevier, Amsterdam.

Hökfelt, T., V. Holets, W. Staines, B. Meister, T. Melander, M. Schallina, M. Schultzberg, J. Freedman, H. Bjorkland, L. Olson, B. Lindh, L.-G. Elfvin, J. Lundberg, J. Lindgren, B. Samuelsson, B. Pernow, L. Terenius, C. Post, B. Everitt, and M. Goldstein (1986) Coexistence of neuronal messengers -an overview. Prog. Brain Res. 68: 33-70.

Jan, L. Y., and Y. N. Jan (1982) Peptidergic transmission in sympathetic ganglia of the frog. J. Physiol. (Lond.) 327: 219-246.
Jensen, R. T., S. W. Jones, K. Folkers, and J. D. Gardner (1984) A synthetic peptide that is a bombesin receptor antagonist. Nature 309 : 61-63.

Jones, S. W. (1985) Muscarinic and peptidergic excitation of bull-frog sympathetic neurones. J. Physiol. (Lond.) 366: 63-87.

Mauborgne, A., O. Lutz, J.-C. Legrand, M. Hamon, and F. Cesselin (1987) Opposite effects of delta and mu opioid receptor agonists on the in vitro release of substance P-like material from the rat spinal cord. J. Neurochem. 48: 529-537.

Miller, R. F., R. A. Zalutsky, and S. C. Massey (1986) A perfused rabbit retina preparation suitable for pharmacological studies. J. Neurosci. Methods 16: 309-322.

Osborne, N. (1984) Substance P in the bovine retina: Localization, identification, release, uptake, and receptor analysis. J. Physiol. (Lond.) 349: 83-93.

Otsuka, M., and S. Konishi (1983) Substance P-the first peptide neurotransmitter? Trends Neurosci. 6: 317-320.

Sagar, S. M., L. Weinstein, J. F. Reinhard, Jr., and J. B. Martin (1983) A comparison of the effects of kainic acid on somatostatin, substance $\mathrm{P}$ and dopamine in the rabbit retina. Brain Res. 278: 109-115.

Vaney, D. I., W. R. Levick, and L. N. Thibos (1981) Rabbit retinal ganglion cells. Exp. Brain Res. 44: 27-33.

Zalutsky, R. A., and R. F. Miller (1985) Physiological actions of neuropeptides on rabbit retinal cells. Invest. Ophthalmol. Vis. Sci. Suppl. 1985: 117.

Zalutsky, R. A., and R. F. Miller (1990) The physiology of somatostatin in the rabbit retina. J. Neurosci. 10:383-393. 